\title{
Vascular allografts are resistant to methicillin-resistant Staphylococcus aureus through indoleamine 2,3- dioxygenase in a murine model
}

\author{
A. Saito, MD, PhD, ${ }^{a}$ N. Motomura, MD, PhD, ${ }^{a}$ K. Kakimi, MD, PhD, ${ }^{b}$ K. Narui, PhD, ${ }^{\mathrm{c}}$ N. Noguchi, $\mathrm{PhD},{ }^{\mathrm{c}} \mathrm{M}$. Sasatsu, PhD, ${ }^{\mathrm{c}}$ \\ K. Kubo, MSc, ${ }^{d}$ Y. Koezuka, PhD, ${ }^{d}$ D. Takai, MD, PhD, ${ }^{e}$ S. Ueha, PhD, ${ }^{f}$ and S. Takamoto, MD, PhD ${ }^{a}$
}

From the Departments of Cardiothoracic Surgery, ${ }^{\mathrm{a}}$ Immunotherapeutics (Medinet), ${ }^{\mathrm{b}}$ Respiratory Medicine, ${ }^{\mathrm{e}}$ and Molecular Preventive Medicine, ${ }^{\mathrm{f}}$ the University of Tokyo Graduate School of Medicine; 'the Department of Microbiology, School of Pharmacy, Tokyo University of Pharmacy and Life Science; and ${ }^{\mathrm{d}}$ the Pharmaceutical Division, Pharmaceutical Research Laboratories, Kirin Brewery Co, Ltd, Tokyo, Japan.

Received for publication Oct 23, 2007; revisions received Dec 14, 2007; accepted for publication Jan 17, 2008.

Address for reprints: Aya Saito, MD, PhD, Division of Cardiac Surgery, London Health Sciences Centre, University Hospital, 339 Windermere Rd, London, Ontario, Canada N6A 5A5 (E-mail: ayasaitou-ths@umin.ac. jp).

J Thorac Cardiovasc Surg 2008;136:159-67 0022-5223/\$34.00

Copyright $\odot 2008$ by The American Association for Thoracic Surgery

doi:10.1016/j.jtcvs.2008.01.006
Objective: Surgical results have shown the superiority of human heart valve and vascular allografts over artificial prostheses when used for the treatment of infectious cardiovascular diseases. However, the mechanism of infection resistance in these allografts has not been determined. In this study the contribution of the inflammatory response after allogeneic transplantation to the antimicrobial mechanism was assessed, focusing on the induction of indoleamine 2,3-dioxygenase, a tryptophanmetabolizing enzyme.

Methods: Aortic transplantation was performed with inbred rats, and aortic allografts, isografts, and control grafts were obtained for the following analyses. The extent of inflammatory-related and indoleamine 2,3-dioxygenase gene expression was measured by means of quantitative reverse transcriptase-polymerase chain reaction, and tryptophan metabolite production in the graft was measured by means of liquid chromatographic/tandem mass spectrometric analysis. The bacteriostatic effect of each graft and tryptophan metabolites was determined by using the methicillin-resistant Staphylococcus aureus proliferation assay.

Results: The inflammatory response, including interferon $\gamma$, tumor necrosis factor $\alpha$, and indoleamine 2,3-dioxygenase gene expression, was significant in the allografts but minimal in the isografts and control grafts. Methicillin-resistant $S$ aureus proliferation was remarkably suppressed when cultured with the allografts but not with the control grafts. Among tryptophan metabolites, the bacteriostatic effect against methicillin-resistant $S$ aureus was remarkable with 3-hydroxykynurenine, with a minimum inhibitory concentration of $32 \mathrm{mg} / \mathrm{L}$. The 3-hydroxykynurenine level in the allografts was 9-fold greater than that in the control grafts.

Conclusion: The bacteriostatic effect of the allografts was acquired by inducing indoleamine 2,3-dioxygenase, which resulted in local production of 3-hydroxykynurenine as an antimicrobial agent. This is the first report to document a mechanism of the allograft's infection-resistant property against methicillin-resistant $S$ aureus growth.

$\mathrm{L}$ ocally advanced prosthetic aortic valve endocarditis often results in a poor clinical outcome with short-term mortality rates of $8 \%$ to $39 \%,{ }^{1-5}$ despite recent progress in antimicrobial therapy and surgical techniques. Gram-positive cocci, such as Staphylococcus aureus, are frequently isolated, and among these, methicillin-resistant $S$ aureus (MRSA) bacteremia is associated with significantly higher mortality rates than other microbes.

Surgical procedures for infective endocarditis treatment usually involve complete resection of the infected area and reconstruction of the defect with valvular prostheses, including mechanical valves, bioprosthetic valves, or allograft heart valves known as homografts. The purported benefits of using homografts are that they do not require adjuvant systemic anticoagulation therapy or immunosuppressive therapy and that they might provide a better clinical outcome for infective cardiovascular disease 


$\begin{array}{ll}\text { Abbreviations and Acronyms } \\ \text { BN } & =\text { Brown Norway } \\ \text { 3-HAA } & =\text {-hydroxyanthranilic acid } \\ 3-\mathrm{HK} & =\text { 3-hydroxykynurenine } \\ \text { IDO } & =\text { indoleamine-2,3-dioxygenase } \\ \text { IFN } & =\text { interferon } \\ \text { Kyn } & =\text { kynurenine } \\ \text { LC-MS/MS } & =\text { liquid chromatography/tandem mass } \\ & \text { spectrometry } \\ \text { LEW } & =\text { Lewis } \\ \text { MH } & =\text { Mueller-Hinton } \\ \text { MRSA } & =\text { methicillin-resistant Staphylococcus aureus } \\ \text { PCR } & =\text { polymerase chain reaction } \\ \text { POD } & =\text { postoperative day } \\ \text { QA } & =\text { quinolinic acid } \\ \text { TNF } & =\text { tumor necrosis factor } \\ \text { Trp } & =\text { tryptophan } \\ \end{array}$

compared with prosthetic devices. Since the 1990s, some retrospective case series have indicated the possible superiority of homograft use for severe infective endocarditis and prosthetic valve endocarditis cases. ${ }^{6-11}$ However, there is no firm consensus of the homografts' potential for antimicrobial capacity because of the absence of randomized controlled studies and scientific evidence supporting this mechanism. ${ }^{12,13}$

Indoleamine 2,3-dioxygenase (IDO) is an interferon (IFN) $\gamma$-induced, rate-limiting enzyme of the L-tryptophan (Trp)-L-kynurenine (Kyn) pathway. ${ }^{14,15}$ IDO is known as one of the most important factors to induce immunologic tolerance by suppressing T-cell proliferation through Trp degeneration and by producing toxic Trp metabolites. ${ }^{16}$ On the other hand, IDO is also known to play a crucial role in mitigating microbial infection by toxic Trp metabolites. ${ }^{17,18}$ Because heart valves and vascular allografts do not usually require blood type or HLA (human leukocyte antigen) type matching between donor and recipient and yet for the most part they survive immunologic attack without adjuvant immunosuppressive therapy, one possible mechanism linking both antimicrobial activity and possible immune tolerance of allografts is the contribution of IDO. In this study we examined how allogeneic vascular grafts resist MRSA infection using a rat subrenal aortic transplantation model. We particularly focused on the role of IDO as a potential contributor to the allograft's antimicrobial activity.

\section{Materials and Methods}

\section{Experimental Model}

Rat in situ vascular allograft transplantation was carried out in the infrarenal position (ie, the infrarenal model). After intraperitoneal injection of pentobarbital ( $5 \mathrm{mg} / 100 \mathrm{~g}$ of body weight), segments of descending thoracic aorta were harvested from Lewis (LEW) rats (RT1A ${ }^{1} \mathrm{~B}^{1}$ Charles River Japan, Inc, Yokohama, Japan) and immediately transplanted to either LEW or Brown Norway (BN) rats $\left(R T 1 A^{n} B^{n}\right)$ after achievement of pentobarbital-induced anesthesia. In the infrarenal model the aortic anastomosis was carried out through a midline laparotomy, and a $1-\mathrm{cm}$ segment graft was anastomosed to the recipient aorta between the renal artery and the terminal aorta in end-to-end fashion with 9-0 nylon interrupted sutures (Bear, Chiba, Japan). In each experiment 4 to 6 rats from each group were sacrificed by means of exsanguination at postoperative days (PODs) 7 or 28, and the grafts were explanted for analysis. Alternatively, aortic graft transplantation to a ventral subcutaneous pocket was applied, and the POD28 samples were obtained. This subcutaneous model is widely accepted as an ectopic transplantation mode $^{19}$ and is also effective in minimizing animal use. No anticoagulants, immunosuppressants, or antibiotics were administered at any time intraoperatively or postoperatively. Initially, grafts from the infrarenal model were used for analysis, including detection of gene expression and the antimicrobial determination assay, because the in situ model was thought to well reflect the clinical use of the allograft. After we confirmed that the grafts obtained from either transplantation model were similar in terms of gene expression pattern and performance against MRSA growth, grafts from the subcutaneous model were used for the rest of the analyses.

In our experiments isograft refers to the explanted grafts obtained from the donor-LEW, recipient-LEW combinations, whereas allograft refers to explanted grafts obtained from donor- LEW, recipient-BN combinations. For the control graft, naive LEW thoracic aortas were used. The experimental protocol was approved by the institutional board of animal care (approval no. 1516T055), and handling and care of the animals were carried out based on the regulations of the Laboratory Animal Center for Experimental Research and the "Guide for the care and use of laboratory animals" prepared by the Institute of Health (National Institute of Health publication no. 85-23, revised in 1985).

\section{Determination of Antibacterial Activity}

MRSA N315 was used for the determination of antibacterial activity. ${ }^{20}$ Allografts obtained from both the subrenal group and the subcutaneous group and control grafts were submitted for analysis. MRSA suspension was prepared with a fresh single colony and saline water. Colony-forming units were calculated by means of the plating of serially diluted bacteria on mannitol salt agar (Eiken Chemical, Tokyo, Japan). The explanted grafts were transferred in $60 \mu \mathrm{L}$ of a 2 -fold concentration of Mueller-Hinton (MH) broth (Oxoid, Hampshire, England) and incubated for 12 hours at $35^{\circ} \mathrm{C}$ for extraction of antibacterial agents from grafts. After centrifugation at $15,000 \mathrm{rpm}$ for 2 minutes, the supernatant was collected and boiled for 2 minutes. The collected supernatant was used as $\times 2$ culture medium for determination of antibacterial activity of the grafts. MRSA solution (approximately 50 colony-forming units of bacteria/10 $\mu \mathrm{L}$ ) and distilled water $(40 \mu \mathrm{L})$ with or without $\operatorname{Trp}$ metabolite was added into the $\times 2$ culture medium or $\times 2 \mathrm{MH}$ medium $(50 \mu \mathrm{L})$. The bacterial mixture $(100 \mu \mathrm{L})$ was incubated at $35^{\circ} \mathrm{C}$. Five microliters of the culture was picked every hour and transferred into $95 \mu \mathrm{L}$ of $\mathrm{MH}$ broth. Immediately, $50 \mu \mathrm{L}$ of the diluted culture was spread onto mannitol salt agar. After incubation for 12 hours at $35^{\circ} \mathrm{C}$, the colony grown on the plate was counted. Bacterial growth in the presence of Trp metabolites was determined by the measure of the optical density at $600 \mathrm{~nm}$ with the microplate photometer SAFIRE (TECAN, Mannedorf, Switzerland). 


\section{RNA Isolation and Real-time Polymerase Chain Reaction}

RNA isolation and real-time polymerase chain reaction (PCR) were performed as previously described. ${ }^{21}$ Total RNA was extracted from tissues with TRIzol reagent (Invitrogen Corp, Carlsbad, Calif), and cDNA was synthesized by a Superscript II Reverse Transcriptase (Invitrogen Corp) by using $2 \mu \mathrm{g}$ of total RNA treated with DNase I (Invitrogen Corp), both according to the manufacturer's instructions. The reaction solution was subjected to real-time PCR reactions on the $\mathrm{M}_{\mathrm{x}} 3000^{\mathrm{TM}}$ real-time PCR system (Stratagene, La Jolla, Calif) for 40 cycles. The PCR condition of each target gene is described in Table 1. Taq Antibody (TAKARA Bio, Inc, Shiga, Japan) or Betaine Monohydrate (Sigma-Aldrich Corp, St Louis, $\mathrm{Mo}$ ) were occasionally added to the PCR reaction solution, as appropriate, to obtain better-quality PCR results. ${ }^{22}$ Emitted fluorescence of SYBR Green (Sigma-Aldrich) for each reaction was measured during the extension phase, and the number of copies was quantified by comparison with standard plasmid obtained by mens of TA cloning (Topo Cloning Kit, Invitrogen Corp). The calculated number of copies was divided by the number of copies of $\beta$-actin and amplified 1000 times for convenience.

\section{Immunohistochemistry}

Four-micrometer-thick frozen sections were fixed for 5 minutes with $10 \%$ neutral buffered formalin. The sections were blocked with $0.5 \%$ goat serum, along with avidin/biotin blocking (Avidin/Biotin Blocking kit; Vector Laboratories, Inc, Burlingame, Calif) before the addition of a primary antibody, per the manufacturer's instructions. The sections were incubated overnight at $4{ }^{\circ} \mathrm{C}$ with goat polyclonal antibody anti-IDO (1:100, Santa Cruz Technology, Santa Cruz, Calif) diluted in $1 \%$ bovine serum albumin. The sections were then reacted with biotinylated anti-rabbit immunoglobulins (1:1000; DAKO, Glostrup, Denmark) for 1 hour at room temperature, followed by incubation with Avidin-Biotinylated enzyme Complex (VECTASTAIN ABC-AP mix; Vector Laboratories, Inc) for 1 hour at room temperature. The immunocomplex was visualized as a brown product after incubating with diaminobenzidine, and counterstaining was done with hematoxylin.

\section{Liquid Chromatographic/Tandem Mass Spectrometric Analysis}

The subcutaneous model grafts were homogenized in distilled water, with a final concentration of $8 \%$ to $10 \%$. Each homogenate was diluted to a concentration of $5 \%$. These were centrifuged for 15 minutes at $13,000 \mathrm{rpm}$ at room temperature, and supernatants were filtered with Ultrafilter (Cosmospin Filter H, $0.45 \mu \mathrm{m}$; Nacalai

TABLE 1. Concentration of Kyn, 3-HK, and 3-HAA in the tissue

\begin{tabular}{lccc}
\hline & Control graft $(\mathbf{n g} / \mathbf{m g})$ & Allograft $(\mathbf{n g} / \mathbf{m g})$ & $\boldsymbol{P}$ value \\
\hline Kyn & $2.16 \pm 0.61$ & $3.88 \pm 2.17$ & ND \\
3-HK & $1.67 \pm 1.22$ & $15.7 \pm 5.11$ & $<.005$ \\
3-HAA & $0.37 \pm 0.12$ & $0.60 \pm 0.09$ & $<.05$ \\
\hline
\end{tabular}

Kyn, Kynurenine; 3-HK, 3-hydroxykynurenine; 3-HAA, 3-hydroxyanthoranilic acid; $N D$, not done.
Tesque, Inc, Kyoto, Japan). After centrifugation, each $180 \mu \mathrm{L}$ of supernatant was mixed with $10 \mu \mathrm{L}$ of water and the internal standard, $200 \mathrm{ng} / \mathrm{mL}$ d-biotin. A $10-\mu \mathrm{L}$ sample preparation was injected for liquid chromatographic/tandem mass spectrometric (LC-MS/MS) analysis. Samples were analyzed by using a hybrid triple quadrupole linear ion-trap API 4000 QTRAP LC-MS/MS system (Applied Biosystems, Inc, Foster City, Calif) with an Agilent 1100 series highperformance liquid chromatography (Agilent Technologies, Inc, Palo Alto, Calif). All spectra were acquired in positive ion mode. The liquid chromatograph was equipped with an Atlantis C18 column $(2.1 \times 50 \mathrm{~mm}, 3-\mu \mathrm{m}$ particle size; Water Corp, Lowell, Mass), and eluents used were as follows: A, $0.025 \mathrm{~mol} / \mathrm{L}$ ammonium acetate; B, acetonitrile (40:60). The flow rate was set to $300 \mu \mathrm{L} / \mathrm{min}$. The ion source was operated in the positive ion mode by using electrospray ionization Turbo Spray ${ }^{\circledR}$ at $700^{\circ} \mathrm{C}$, and analyses were performed by using enhanced product ion scanning and the multiple-reaction monitoring mode. Signals of characteristic ions for each Trp metabolite and the internal standard (d-biotin) were described as follows: Kyn (209.00 $\rightarrow$ 94.00), 3-hydroxykynurenine (3-HK; $225.10 \rightarrow$ 110.20), 3-hydroxyanthranilic acid (3-HAA; $154.10 \rightarrow 80.10)$, and d-biotin $(245.15 \rightarrow 97.00)$. Collision energies were optimized as $39 \mathrm{eV}$ for Kyn, $23 \mathrm{eV}$ for $3-\mathrm{HK}$, and 39 $\mathrm{eV}$ for 3-HAA, respectively.

\section{Statistical Analysis}

Results were presented as means \pm standard error of the mean. Comparisons were made by using the Student $t$ test or analysis of variance with the Bonferroni/Dunn post-hoc test, as appropriate.

\section{Results \\ MRSA Growth Suppression}

Initially, we transplanted LEW rat thoracic aortas into BN rats using both the infrarenal and subcutaneus models. The grafts were explanted at POD28 and submitted to the antimicrobial determination assay by using MRSA, in which the grafts were incubated in MH broth and the supernatant of the broth was applied to cultivation of MRSA on mannitol salt agar to estimate the extent of MRSA proliferation. Bacterial growth was determined based on the optical density at $600 \mathrm{~nm}$ for quantification. No MRSA proliferation was observed for the first 7 hours in all the assays, including the infrarenal allograft, subcutaneus allograft, and control graft (naive LEW aorta) assays (Figure 1, A). The proliferation curve for control grafts started to steeply rise after 7 hours of cultivation, whereas the proliferation curve for the allografts still showed very low proliferation. At the end of the assay, after 10 hours of cultivation, MRSA colony count was 59,360.0 $\pm 11,622.0$ for the control graft assay, whereas it was 20,146.7 \pm 2417.4 for the infrarenal allograft assay and 15,686.7 \pm 2523.5 for the subcutaneous allograft assay $(P<.01$ for both allograft groups vs the control group). The extent of MRSA growth suppression in the infrarenal allograft and the subcutaneous allograft was not statistically different. The difference in MRSA proliferation between the allograft assay and control graft assay could be visualized by the number of colonies on the mannitol salt agar (Figure 1,B). The top 

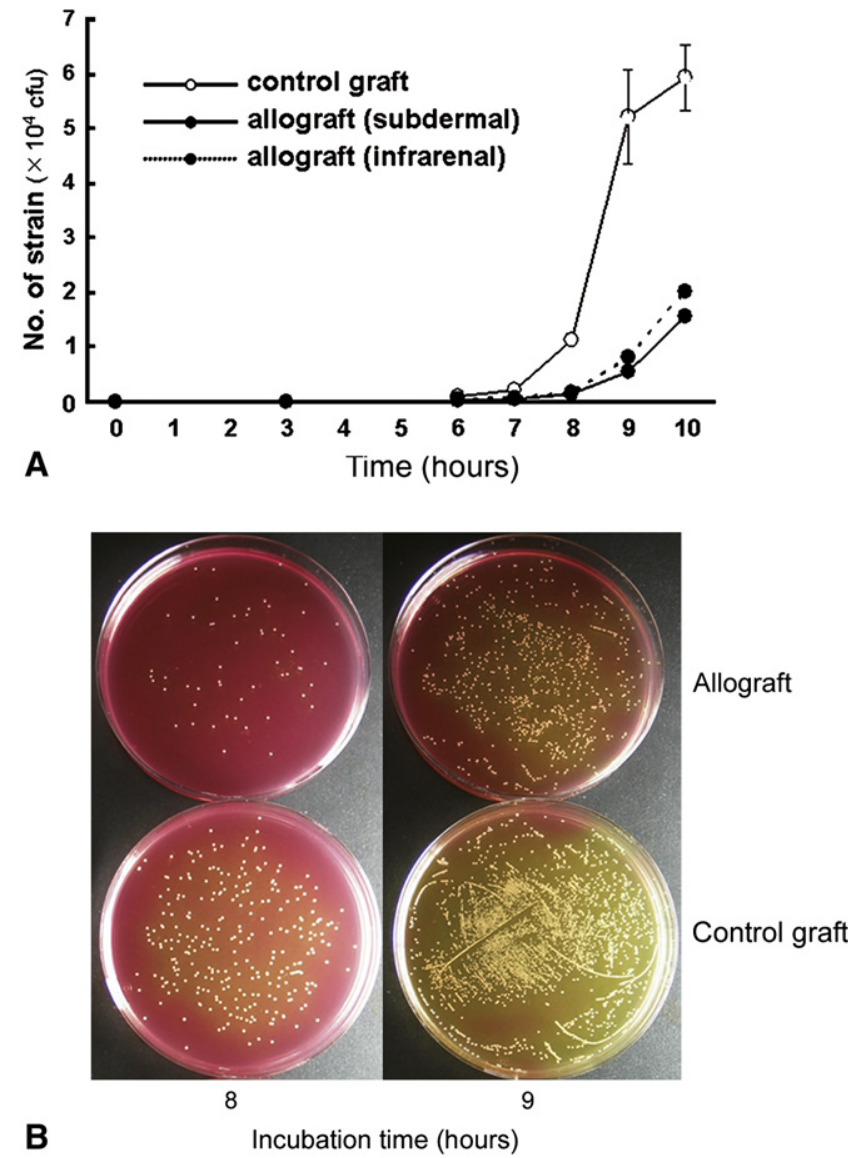

Figure 1. Antimicrobial activity of the allografts and control grafts against methicillin-resistant Staphylococcus aureus (MRSA) proliferation in vitro. The allografts were obtained from either the infrarenal or subcutaneous model at postoperative day 28 . The explanted grafts and control grafts were submitted to MuellerHinton broth, respectively, and the suspension was applied to mannitol salt agar every hour to estimate the difference in MRSA proliferation. A, MRSA colony count is expressed in the graph by time course. MRSA proliferation was remarkable when cultured with control grafts (open circles), whereas allografts obtained by means of subdermal (open squares) or infrarenal (open diamonds) transplantation presented MRSA growth suppression. B, MRSA proliferation was visualized on the mannitol salt agar 8 hours (left) and 9 hours (right) after incubation. The MRSA proliferation was remarkably suppressed for allografts compared with that seen in control grafts.

2 plates of Figure 1, $B$, demonstrate the results of the infrarenal allograft assay, and the lower 2 plates show the results of the control graft assay (left, 8 hours of cultivation; right, 9 hours of cultivation). In summary, these series of assays revealed that the rat aortic grafts acquired antimicrobial activity to MRSA after allogeneic transplantation. We also showed that the allograft after infrarenal transplantation and subcutaneus transplantation did not differ in antimicrobial activity.
In the next series of experiments, we examined induction of the inflammatory response followed by IDO expression as a possible contributor to the antimicrobial activity presented by allografts.

\section{Inflammatory Cytokines and IDO Induction After Allogeneic Transplantation}

In this section we tried to verify the induction of a specific inflammatory response after allograft transplantation through IDO expression, which we hypothesized is a key factor for antimicrobial activity of the allografts. To that end, we assessed gene expression for tumor necrosis factor (TNF) $\alpha$, IFN- $\gamma$, and IDO in the explanted grafts. Gene expression in the isografts was assessed for comparison to estimate the extent of non-specific surgical manipulation-derived inflammatory response. By using the infrarenal transplantation model, the isografts and the allografts obtained at POD7 and POD28 were submitted to quantitative reverse transcriptasePCR for analysis together with the control grafts (Figure 2, $A$ ). TNF- $\alpha$ gene expression was remarkably increased in the allografts, whereas isografts showed significantly lower gene expression, and the level in the allografts was 6-fold greater than that in the isografts at POD28. For IFN- $\gamma$ gene expression, gene expression also increased in the allografts, and the expression level was 20-fold greater in the allografts than in the isografts at POD28 (Figure 2, B). IDO, expressed downstream of IFN- $\gamma$ gene expression, was also remarkably increased in the allografts, and its level at POD28 was almost 60 -fold greater in the allografts than in the isografts (Figure 2,C). In the control grafts all of the target genes (TNF- $\alpha$, IFN- $\gamma$, and IDO) were detected at very low levels, and there was no statistical difference for each gene expression between control grafts and isografts at any time point. The inflammatory response and IDO expression in the allografts was also confirmed by means of histopathologic examination (Figure 3). Hematoxylin and eosin staining of the explanted allograft revealed gross inflammatory cell infiltration in the adventitia and occasionally in the intima (Figure 3, B). IDO was detected by means of immunohistochemical staining in the same distribution as the inflammatory cells in the intima and the adventitia (Figure 3, $C)$. These results showed that a specific inflammatory response was induced within the grafts accompanying IDO gene and protein expression, suggesting that IDO participates in the allograft's antimicrobial activity at the site of allogeneic transplantation.

\section{MRSA Proliferation Suppression by the Trp Metabolites}

In the previous sections we observed that rat aortic allografts acquired antimicrobial activity through allogeneic transplantation and that the allografts presented IDO gene and protein expression after IFN- $\gamma$ induction. Because IDO expression has been viewed as crucial for regulating inflammatory 

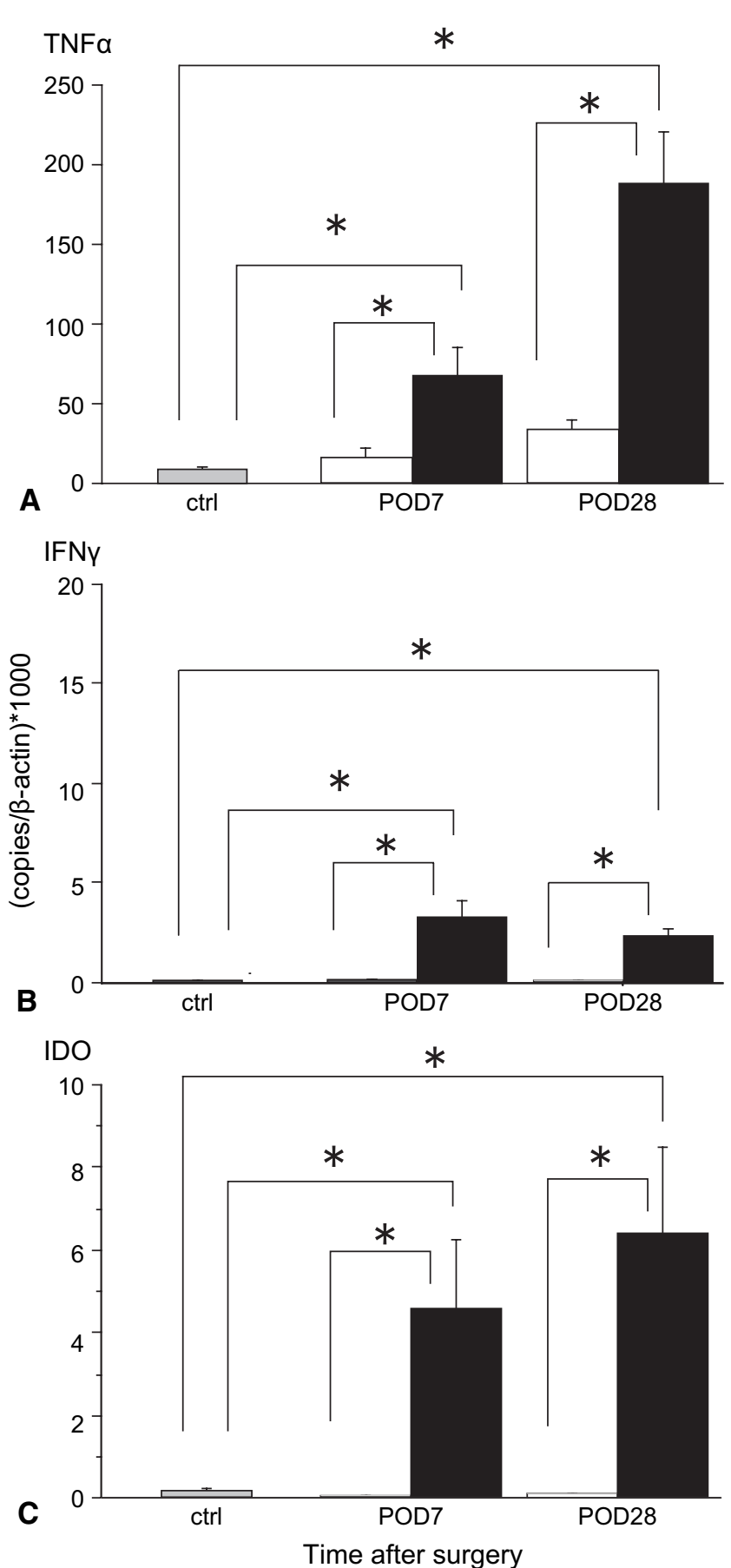

isograft allograft control grafts(ctrl)

Figure 2. Results of mRNA expression for tumor necrosis factor (TNF) $\alpha$ (A), interferon (IFN) $\gamma(\mathrm{B})$, and indoleamine-2,3-dioxygenase $(I D O ; C)$ in the explanted grafts by means of quantitative reverse transcriptase-polymerase chain reaction. The expression level was compared between control grafts (ctrl, small dot bar), allografts (closed bar), and isografts (open bar) at postoperative days (POD) 7 and 28, and the control grafts (without transplantation manipulation) were also examined. TNF- $\alpha$ and IFN- $\gamma$ levels increased over time in the allografts, and the difference between response through toxic metabolites of Trp, ${ }^{17,18}$ we investigated the antimicrobial activity of Trp and its major metabolites against MRSA proliferation. The metabolites included Kyn, 3-HK, anthoranilic acid, 3-HAA, and quinolinic acid (QA). These substances were prepared in series of concentrations of 256,32 , and $8 \mu \mathrm{g} / \mathrm{mL}$.

The results from these series of assays are shown in Figure 4. MRSA started proliferating after 10 hours of cultivation under the appearance of Trp at any concentration, and the same proliferation pattern was obtained from the assay with Kyn (Figure 4, $A$ and $B$ ). These results implied that Trp and Kyn had no antimicrobial activity. From Figure 4, $C$, MRSA growth suppression was clearly detected under the influence of $3-\mathrm{HK}$ at 256 and $32 \mu \mathrm{g} / \mathrm{mL}$, which lasted at least 18 hours after cultivation. The assay with anthoranilic acid and 3-HAA also showed mild growth suppression only at $256 \mu \mathrm{g} / \mathrm{mL}$ (Figure $4, D$ and $E$ ). QA also had just a mild temporal effect (Figure $4, F$ ). These series of results indicate that some of the Trp metabolites are toxic to MRSA, and among them, 3-HK has relatively strong antimicrobial activity.

In all of the previous experiments, we have observed antimicrobial activity in the allografts and have confirmed IDO expression in the allografts as a key factor of this antimicrobial activity. For a further understanding of this mechanism, we investigated the influence of each Trp metabolite on MRSA growth, which proved that 3-HK has moderate bacteriotoxicity. The next issue to be clarified was whether 3 -HK is actually produced at the site of allogeneic transplantation.

\section{Production of Trp Metabolites in the Grafts}

In the next series of experiments, we investigated whether IDO is active as an enzyme in the allografts, thus producing 3-HK, which has a bacteriotoxic effect against MRSA. The homogenate of the allografts and control grafts was subjected to LC-MS/MS analysis for detection and quantification of Kyn, 3-HK, and 3-HAA within the graft tissue. The quantification was performed by calculating in comparison to the standard area for $10 \mathrm{ng} / \mathrm{mg}$ of each substance. The results for Kyn can be seen in the top 3 graphs of Figure 5, A, with the standard area curve on the left, control in the middle, and allograft on the right. The peak area for Kyn in the allograft tissue was greater than that in the control graft tissue, as was the peak area for 3-HAA (Figure 5, $C)$. The peak area for $3-\mathrm{HK}$, the key substance among the Trp metabolites, was also detected in the allograft tissue, and its level was significantly greater in the allograft tissue than in the control tissue (Figure 5, B). The calculated

the allografts and isografts was remarkable at POD28. IDO expression became apparent in the allografts at POD7, whereas almost no expression was observed in the isografts. ${ }^{*} P<.05$. 


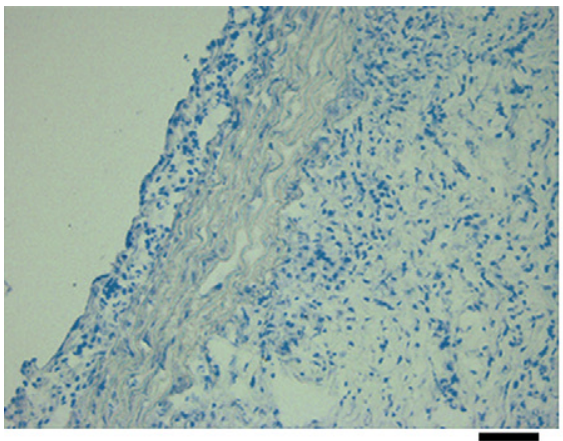

A
Control staining

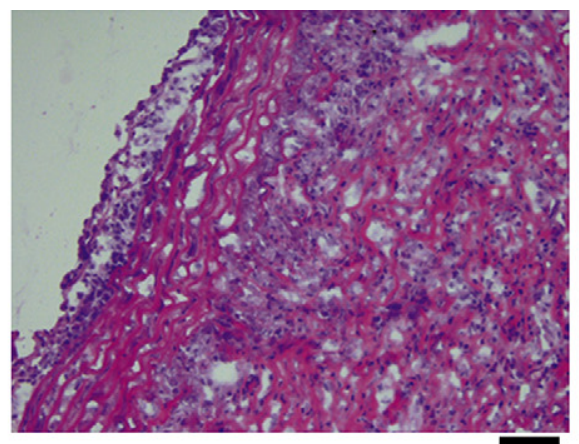

B Hematoxylin Eosin

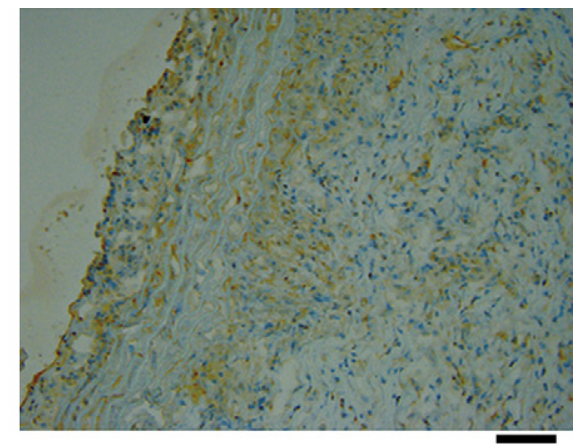

c
IDO (DAB)

Figure 3. Histologic detection of inflammatory changes and IDO expression in the allograft. A, Control; B, hematoxylin and eosin stain; $C$, immunohistochemical staining for indoleamine-2,3-dioxygenase (IDO). There was a remarkable inflammatory cell infiltration in the intima and adventitia (B), where IDO was widely expressed. Bars indicate $50 \mu \mathrm{m}$. DAB, Diaminobenzidine.

amount of each substance is shown in Table 1. The allograft tissue contained approximately 9 times greater 3 -HK levels than the control tissue versus 1.5 times for 3-HAA and al- most the same amount for Kyn. Therefore 3-HK was actually produced in the allografts in much greater levels than in the control grafts, suggesting that IDO induction, along $\multimap 8 \mu \mathrm{g} / \mathrm{mL} \longrightarrow-32 \mu \mathrm{g} / \mathrm{mL} \longrightarrow 256 \mu \mathrm{g} / \mathrm{mL}$
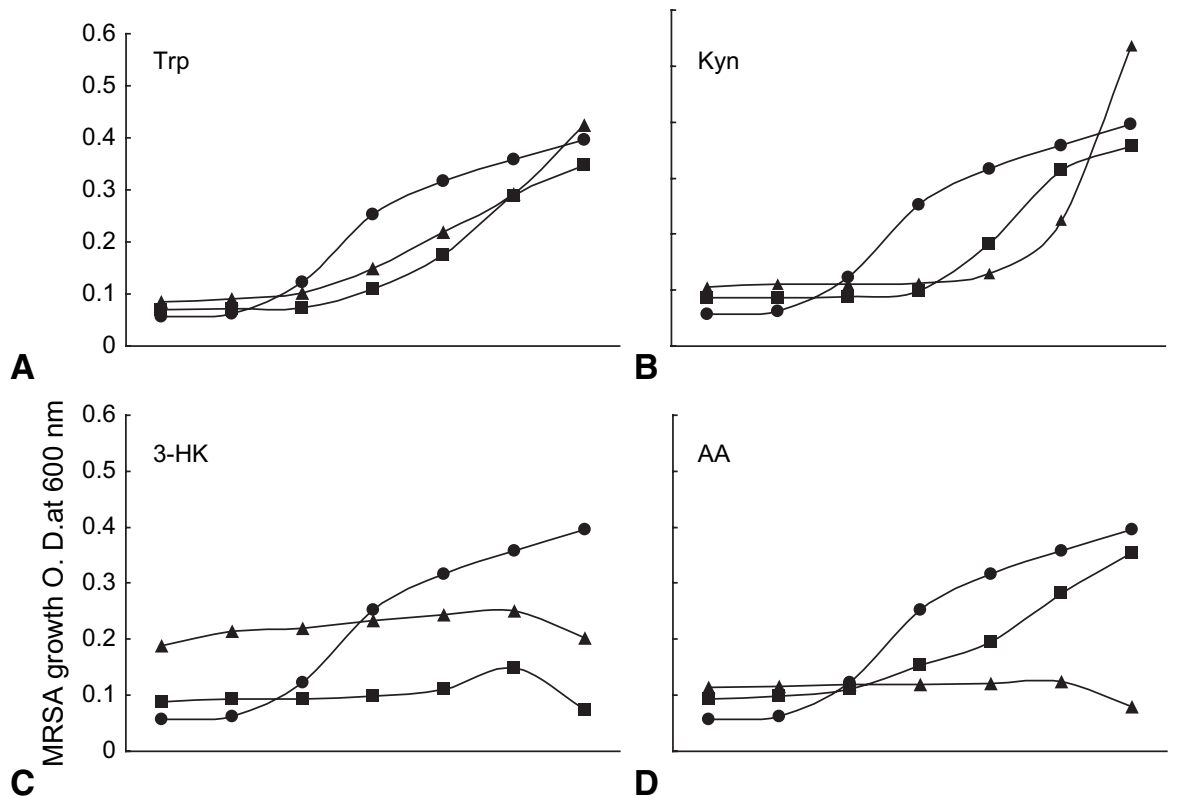

AA

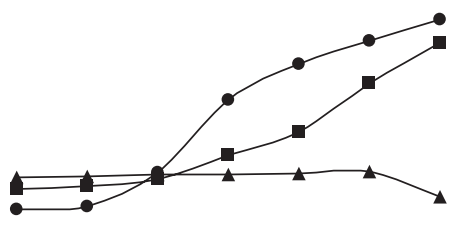

D

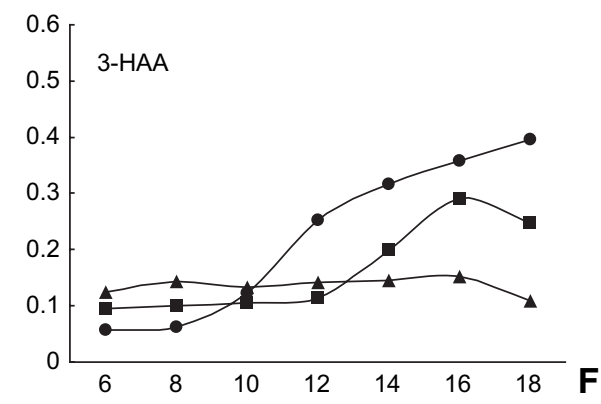

QA

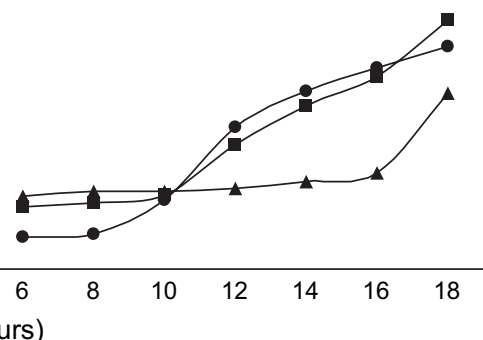

Figure 4. Antimicrobial activity of tryptophan (Trp; Panel A) and its metabolite against methicillin-resistant Staphylococcus aureus (MRSA) at different concentrations $(256 \mu \mathrm{g} / \mathrm{mL}$, closed circles; $32 \mu \mathrm{g} / \mathrm{mL}$, closed squares; $8 \mu \mathrm{g} /$ $\mathrm{mL}$, closed triangles). Bacterial growth was determined based on the optical density (0.D.) at $600 \mathrm{~nm}$ by a using microplate photometer. Among those substances, 3-hydroxykynurenine (3-HK) showed relatively strong suppression on MRSA growth at a concentration of $32 \mu \mathrm{g} / \mathrm{mL}$ (C), and 3-hydroxyanthoranilic acid (3-HAA; E) showed a very mild antimicrobial effect at $256 \mu \mathrm{g} / \mathrm{mL}$. Kyn (Panel B), Kynurenine; 3-HK (Panel C), 3-Hydroxykynurenine; 3-HAA (Panel E), 3-Hydroxyanthoranilic acid; $A A$ (Panel D), anthoranilic acid; $Q A$ (Panel F), quinolinic acid. 

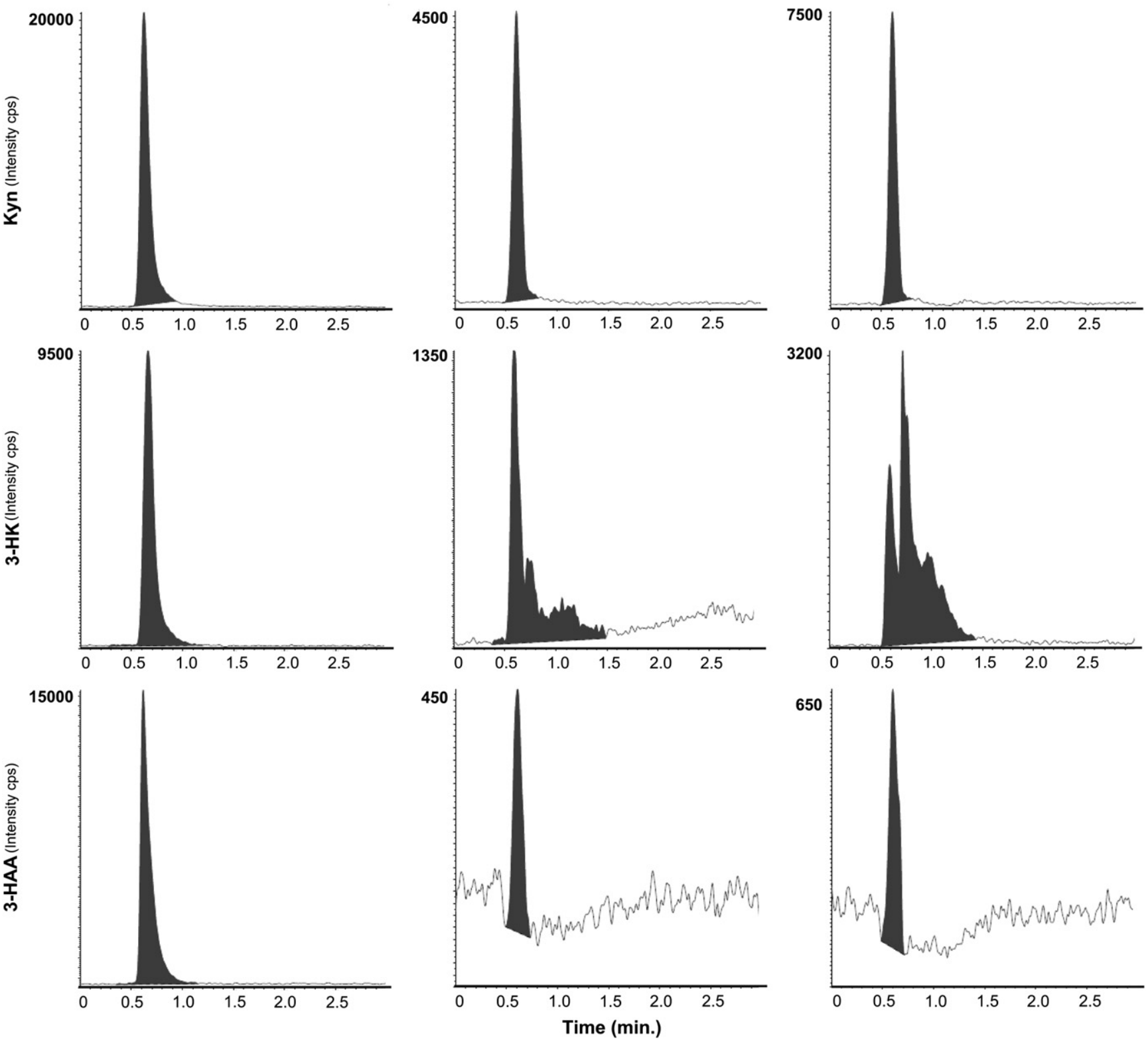

Figure 5. Kynurenine (Kyn), 3-hydroxykynurenine (3-HK), and 3-hydroxyanthoranilic acid (3-HAA) content in the grafts by means of liquid chromatographic/tandem mass spectrometric (LC-MS/MS) analysis. The grafts, either explanted or control, were homogenized in distilled water, and the supernatant was submitted to LC-MS/MS. By using the standard curve, the content was quantified by measuring the identical peak area and calculating in accordance with the standard curve. Among tryptophan (Trp) and its metabolites, 3-HK was detected in the grafts, with a significant difference found between the allografts and the control grafts (see also Table 1).

with 3-HK production, could contribute to the allograft's tolerance against MRSA infection.

\section{Discussion}

The main findings of this study were that the animal tissue can present antimicrobial activity against MRSA after allo- geneic transplantation in association with the production of 3-HK, a Trp metabolite by IDO. This concept could explain the clinically observed benefit of using human heart valve and vascular allografts in patients with infective endocarditis and infective aortic aneurysms, especially MRSA-related infection; that organism is one of the most 
problematic bacteria involved in infective endocarditis and prosthetic valve endocarditis in the clinical arena.

Our initial series of antimicrobial activity assays clearly showed remarkable MRSA growth suppression in the allografts, and this was not observed in the control graft assays. The MRSA assay that we adopted was our original model, which allowed us to measure the extent of MRSA growth under the influence of byproducts after allogeneic transplantation in the exudate broth of the graft, which was stable against heat (data not shown). The fact that boiled exudate maintained antimicrobial activity supports the concept that the key element is a heat-stable non-protein substance compatible with a Trp metabolite. To our knowledge, our report is the first to prove that homologous grafts acquire antimicrobial capacity through allogeneic transplantation, where the influence of a specific inflammatory response exists, through an IFN- $\gamma$-mediated immunoregulated response.

In this study we have drawn attention to IDO activity, derived from the IFN- $\gamma$-mediated specific immune response, as a possible antimicrobial mechanism that could link to the immunogenicity of allografts. IDO has been recognized as a key enzyme for regulating the inflammatory response at the site of infection by producing toxic Trp metabolites and by consuming an indispensable structural component of microorganisms. ${ }^{17,18,23-26}$ Because there has been no previous report showing the direct antimicrobial effects of specific Trp metabolites, we studied these substances in detail, focusing on their effects against MRSA. We observed a strong bacteriostatic effect of 3-HK, which was isolated from the allograft by means of LC-MS/MS analysis. A recent report has shown that Trp metabolites are effective in the treatment of $\mathrm{T}_{\mathrm{H}} 1$-mediated autoimmune disease by inhibiting autoreactive $\mathrm{T}_{\mathrm{H}} 1$ cells. ${ }^{27}$ Other reports have also shown that 3 -HK and QA have neurotoxicity among the Kyn pathway, a major Trp-metabolizing pathway led by IDO activity. ${ }^{28-30}$ This neurotoxicity have been described partially as a result of conversion of 3-HK and QA to reactive oxygen species that initiate apoptosis, an effect that would be reduced by radical scavengers. ${ }^{29}$ The antimicrobial effect of 3 -HK noted in this study might be derived, in part, from the similar endogenous oxidative stress generation of 3-HK and QA.

Recent experiments on infection and IDO have shown that the endogenous protection mechanism of living tissue can induce IDO in the context of inflammatory response against infectious stimuli, thus exhibiting antimicrobial activity. ${ }^{17,18,23-26}$ In the context of these previous reports, our experiments highlight the underlying mechanism of allograft resistance to infectious cardiovascular disease. Moreover, the benefit of surgical treatment with allograft heart valves for severe infective endocarditis and prosthetic valve endocarditis of the aortic root must include sufficient debridement and direct irrigation of the infected area, the proper 3-dimensional reconstruction of destructive lesions, and the prevention of local bacterial growth by 3-HK, a key product of IDO activity triggered by a local IFN- $\gamma$-mediated immune response.

Nonetheless, these studies are still preliminary, and several issues need to be clarified in the future. These include convincingly proving the existence of IDO interference to the allograft antimicrobial effect by applying an IDO inhibitor (eg, 1-methyltryptophan) or using IDO knockout animals. Additional experiments need to investigate the precise mechanisms of 3-HK, 3-HAA, and QA on the microorganisms and to determine the magnitude of IDO's role among all the possible anti-infectious factors. Furthermore, the use of tissueengineered autografts for infectious cardiovascular disease should be investigated because these grafts also have the potential of inducing IFN- $\gamma$-related inflammation, with an expectation of similar antimicrobial activity as seen in allografts. In addition to antimicrobial activity, IDO might interfere with immunologic tolerance of homografts, and experiments are underway in our laboratory to investigate this.

In summary, our results suggest that the induction of IDO in the context of inflammatory responses to allogeneic transplantation makes the allografts resistant to bacterial infection by producing the toxic Trp metabolite 3-HK. Together with the immunologic tolerance effect by IDO, it is likely that the allogeneic immune response supports both of the beneficial processes of the host/pathogen and host/graft interface by inducing IDO in the allograft tissue. In addition, the agents that can induce 3 -HK might have potential therapeutic value.

We thank Professor Richard J. Novick for help with editorial revisions.

\section{References}

1. Hasbun R, Vikram HR, Barakat LA, Buenconsejo J, Quagliarello VJ. Complicated left-sided native valve endocarditis in adults: risk classification for mortality. JAMA. 2003;289:1933-40.

2. Chu VH, Cabell CH, Benjamin DK Jr, Kuniholm EF, Fowler VG Jr, Engemann J, et al. Early predictors of in-hospital death in infective endocarditis. Circulation. 2004;109:1745-9.

3. Cabell CH, Abrutyn E, Fowler VG Jr, Hoen B, Miro JM, Corey GR, et al. Use of surgery in patients with native valve infective endocarditis: results from the International Collaboration on Endocarditis Merged Database. Am Heart J. 2005; 150:1092-8.

4. Anguera I, Miro JM, San Roman JA, de Alarcon A, Anguita M, Almirante B, et al. Periannular complications in infective endocarditis involving prosthetic aortic valves. Am J Cardiol. 2006;98:1261-8.

5. Anguera I, Miro JM, Evangelista A, Cabell CH, San Roman JA, Vilacosta I, et al. Periannular complications in infective endocarditis involving native aortic valves. Am J Cardiol. 2006;98:1254-60.

6. Yankah AC, Pasic M, Klose H, Siniawski H, Weng Y, Hetzer R. Homograft reconstruction of the aortic root for endocarditis with periannular abscess: a 17-year study. Eur J Cardiothorac Surg. 2005;28:69-75.

7. Sabik JF, Lytle BW, Blackstone EH, Marullo AG, Pettersson GB, Cosgrove DM. Aortic root replacement with cryopreserved allograft for prosthetic valve endocarditis. Ann Thorac Surg. 2002;74:650-9.

8. Grinda JM, Mainardi JL, D'Attellis N, Bricourt MO, Berrebi A, Fabiani JN, et al. Cryopreserved aortic viable homograft for active aortic endocarditis. Ann Thorac Surg. 2005;79:767-71.

9. Vogt PR, von Segesser LK, Jenni R, Niederhauser U, Genoni M, Kunzli A, et al. Emergency surgery for acute infective aortic valve endocarditis: performance of cryopreserved homografts and mode of failure. Eur J Cardiothorac Surg. 1997;11:53-61. 
10. Leyh RG, Knobloch K, Hagl C, Ruhparwar A, Fischer S, Kofidis T, et al. Replacement of the aortic root for acute prosthetic valve endocarditis: prosthetic composite versus aortic allograft root replacement. J Thorac Cardiovasc Surg. 2004;127:1416-20.

11. Haydock D, Barratt-Boyes B, Macedo T, Kirklin JW, Blackstone E. Aortic valve replacement for active infectious endocarditis in 108 patients. A comparison of freehand allograft valves with mechanical prostheses and bioprostheses. J Thorac Cardiovasc Surg. 1992;103:130-9.

12. Eichinger WB, Goppel G, Mendler N, Mattes J, Lankes E, Botzenhardt $\mathrm{F}$, et al. In-vivo bacterial adherence to intracardiac prosthetic materials: a new experimental model. J Heart Valve Dis. 2002; 11:438-46.

13. Rowe NM, Impellizzeri P, Vaynblat M, Lawson NM, Kim YD, Sierra M, et al. Studies in thoracic aortic graft infections: the development of a porcine model and a comparison of collagen-impregnated Dacron grafts and cryopreserved allografts. J Thorac Cardiovasc Surg. 1999;118:857-65.

14. Munn DH, Zhou M, Attwood JT, Bondarev I, Conway SJ, Marshall B, et al. Prevention of allogeneic fetal rejection by tryptophan catabolism. Science. 1998;281:1191-3.

15. Mellor AL, Munn DH. IDO expression by dendritic cells: tolerance and tryptophan catabolism. Nat Rev Immunol. 2004;4:762-74.

16. Bauer TM, Jiga LP, Chuang JJ, Randazzo M, Opelz G, Terness P. Studying the immunosuppressive role of indoleamine 2,3-dioxygenase: tryptophan metabolites suppress rat allogeneic $\mathrm{T}$-cell responses in vitro and in vivo. Transpl Int. 2005; 18:95-100.

17. Silva NM, Rodrigues CV, Santoro MM, Reis LF, Alvarez-Leite JI, Gazzinelli RT. Expression of indoleamine 2,3-dioxygenase, tryptophan degradation, and kynurenine formation during in vivo infection with Toxoplasma gondii: induction by endogenous gamma interferon and requirement of interferon regulatory factor 1. Infect Immun. 2002;70: 859-68.

18. Bozza S, Fallarino F, Pitzurra L, Zelante T, Montagnoli C, Bellocchio S, et al. A crucial role for tryptophan catabolism at the host/Candida albicans interface. J Immunol. 2005;174:2910-8.

19. Webb CL, Nguyen NM, Schoen FJ, Levy RJ. Calcification of allograft aortic wall in a rat subdermal model. Pathophysiology and inhibition by Al3 + and aminodiphosphonate preincubations. Am J Pathol. 1992;141: 487-96.
20. Kuroda M, Ohta T, Uchiyama I, Baba T, Yuzawa H, Kobayashi I, et al. Whole genome sequencing of methicillin-resistant Staphylococcus aureus. Lancet. 2001;357:1225-40.

21. Saito A, Motomura N, Kakimi K, Ono M, Takai D, Sumida S, et al. Cryopreservation does not alter the allogenicity and development of vasculopathy in post-transplant rat aortas. Cryobiology. 2006;52:251-60.

22. Henke W, Herdel K, Jung K, Schnorr D, Loening SA. Betaine improves the PCR amplification of GC-rich DNA sequences. Nucleic Acids Res. 1997;25:3957-8.

23. Pantoja LG, Miller RD, Ramirez JA, Molestina RE, Summersgill JT. Inhibition of Chlamydia pneumoniae replication in human aortic smooth muscle cells by gamma interferon-induced indoleamine 2, 3-dioxygenase activity. Infect Immun. 2000;68:6478-81.

24. Fujigaki S, Saito K, Takemura M, Maekawa N, Yamada Y, Wada H, et al. L-tryptophan-L-kynurenine pathway metabolism accelerated by Toxoplasma gondii infection is abolished in gamma interferon-gene-deficient mice: cross-regulation between inducible nitric oxide synthase and indoleamine-2,3-dioxygenase. Infect Immun. 2002;70:779-86.

25. Hucke C, MacKenzie CR, Adjogble KD, Takikawa O, Daubener W. Nitric oxide-mediated regulation of gamma interferon-induced bacteriostasis: inhibition and degradation of human indoleamine 2,3-dioxygenase. Infect Immun. 2004;72:2723-30.

26. Mackler AM, Barber EM, Takikawa O, Pollard JW. Indoleamine 2,3-dioxygenase is regulated by IFN-gamma in the mouse placenta during Listeria monocytogenes infection. J Immunol. 2003;170:823-30.

27. Platten M, Ho PP, Youssef S, Fontoura P, Garren H, Hur EM, et al. Treatment of autoimmune neuroinflammation with a synthetic tryptophan metabolite. Science. 2005;310:850-5.

28. Okuda S, Nishiyama N, Saito H, Katsuki H. 3-Hydroxykynurenine, an endogenous oxidative stress generator, causes neuronal cell death with apoptotic features and region selectivity. J Neurochem. 1998;70: 299-307.

29. Chiarugi A, Meli E, Moroni F. Similarities and differences in the neuronal death processes activated by $3 \mathrm{OH}-\mathrm{kynurenine}$ and quinolinic acid. J Neurochem. 2001;77:1310-8.

30. Stone TW, Darlington LG. Endogenous kynurenines as targets for drug discovery and development. Nat Rev Drug Discov. 2002;1:609-20. 